Case Report

\title{
A case of spontaneous pneumothorax, pneumomediastinum and subcutaneous emphysema in Pneumocystis jirovecii pneumonia complicating HIV
}

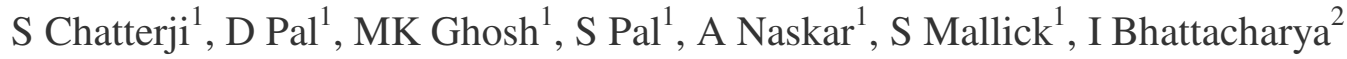 \\ Sri Lankan Journal of Infectious Diseases 2015 Vol.5 (1):22-27 \\ DOI: http://dx.doi.org/10.4038/sljid.v5i1.7569
}

\begin{abstract}
:
Spontaneous pneumothorax and subcutaneous emphysema are well-known but less common complications of pneumocystis pneumonia, whereas pnuemomediastinum is rare. We had a patient known to be HIV seropositive, who developed pneumocystis jirovecii pneumonia. During the course of treatment, he developed spontaneous pneumothorax, pneumomediastinum and subcutaneous emphysema. He had no predisposing factors known to cause this complication.
\end{abstract}

Key words: Pneumomediastinum, pneumothorax, subcutaneous emphysema, Pneumocystis jirovecii,

\section{Introduction:} AIDS.

Pneumocystis jirovecii is an opportunistic fungus found ubiquitously. It causes interstitial pneumonia in immunocompromised hosts. It is acquired by inhalation and can cause fatal disease if not recognized early, especially in persons with some form of immunodeficiency. In severe disease, spontaneous pneumothorax and subcutaneous emphysema can occur but pnuemomediastinum occurs only rarely. ${ }^{1}$ Doppman et. $\mathrm{al}^{2}$ in a review of atypical radiographic features in 30 cases of pneumocystis pneumonia specifically noted a total absence of spontaneous pneumothorax in adults. Recent reports ${ }^{3,4}$ however have shown that Pneumocystis jirovecii pneumonia can cause lung tissue destruction and spontaneous pneumothorax in patients with the acquired immunodeficiency syndrome (AIDS).

\section{Case Report:}

A 36 year old gentleman was suffering from low grade, intermittent fever with loose motions for one month. It was associated with non-productive cough and exertional shortness of breath for fifteen days. His breathlessness was insidious in onset and progressive in nature. He received anti-tubercular therapy for pulmonary tuberculosis seven years back and was found to be seropositive for HIV-1 (by rapid antibody card test, test kit batch no - 4000012651, date of expiry - 28.05.2015) three weeks before the present illness. He was a non-smoker and height was $162 \mathrm{~cm}$.

On examination, the patient was alert, conscious and had evidence of oropharyngeal candidiasis. He was tachypnoeic (respiratory rate 40/min), with a heart rate of 104/minute and normal blood

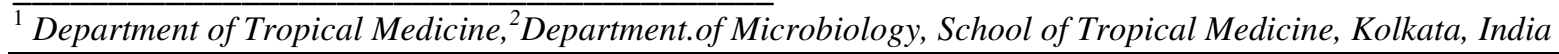
Address for correspondence: Dr.Dipankar Pal, Department of Tropical Medicine, School of Tropical Medicine, Kolkata, India.E-mail : dipankarpal.2009@gmail.com Phone-91-9432113713 
pressure, single breath count was 8 , cyanosis was present and $\mathrm{SpO} 2$ was $90 \%$ at rest without oxygen. Except a small area of diminished breath sounds in the right infra-axillary area, the chest examination was unremarkable. Chest $\mathrm{x}$-ray done 15 days previously was normal. However, the $\mathrm{x}$-ray done on admission revealed right lower zone infiltrations. No cavity/cyst was apparent. His sputum grew Streptococcus pyogenes but acid fast bacilli were absent. Blood CD4 count was 91 cells $/ \mu \mathrm{L}$. He was given moist oxygen inhalation, oral amoxicillin for streptococcal infection and fluconazole for oropharyngeal candidiasis.

The clinical presentation and a low CD4 count in an HIV infected subject, led to the suspicion of Pneumocystis jirovecii pneumonia (PJP). Sputum induction done with hypertonic saline nebulization showed the presence of Pneumocystis jirovecii cysts in Giemsa stained smear under microscope.(Fig.1)

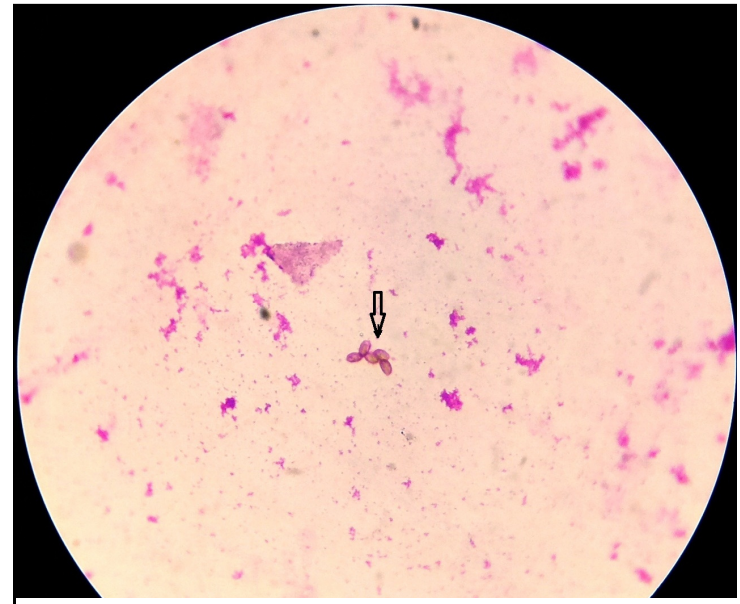

Figure. 1: Sputum smear showing Pneumocystis jirovecii cysts (black arrow) in Giemsa stained smear 100X magnification.

He was immediately shifted to the critical care unit and closely monitored. Repeat $\mathrm{x}$-ray revealed subcutaneous emphysema and right sided small pneumothorax with pneumomediastinum (Fig.2). Contrast enhanced CT (CECT) thorax confirmed the presence of pneumomediastinum and also revealed the presence of some cystic lesions and bronchiectatic changes in both lungs with ground glass opacity (Fig.3,4).

The patient was kept on conservative treatment with close monitoring. His subcutaneous emphysema, pneumothorax and

pneumomediastinum resolved in about two weeks. $\mathrm{He}$ was returned to the general ward. Antiretroviral therapy (ART) was initiated and he was discharged on ART and secondary prophylaxis for
Treatment with co-trimoxazole and prednisolone was started in the recommended doses (trimethoprim $15 \mathrm{mg} / \mathrm{kg} /$ day). ${ }^{5}$ He started to improve gradually. Cyanosis was absent on day three and he became ambulatory.

On day ten of admission, he complained of sudden increase in shortness of breath started at rest and became cyanotic again. Subcutaneous emphysema was noted on both sides of neck. Trachea and apex beat were normal in position and there was slight diminution of vesicular breath sound bilaterally.

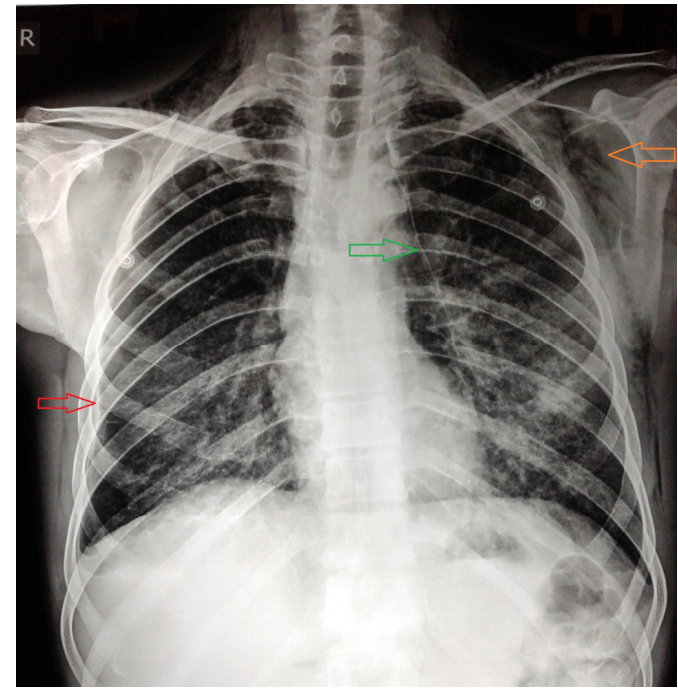

Figure. 2: Chest $x$-ray showing

a) Collapsed lung border (red arrow),

b) Subcutaneous emphysema (yellow arrow)

c) Pneumomediastinum (green arrow). 
Pneumocystis jirovecii with co-trimoxazole. The major events in the process of disease evolution are depicted in Fig 5.

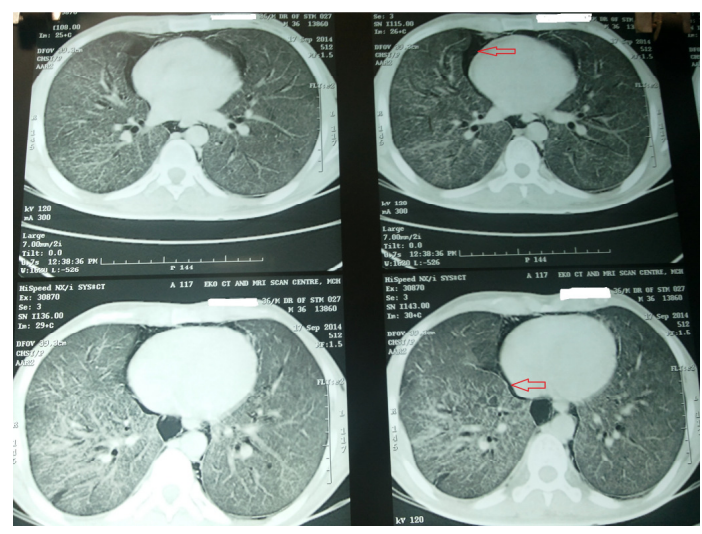

Figure. 3: CECT thorax showing ground glass opacity and pneumomediastinum (red arrows)

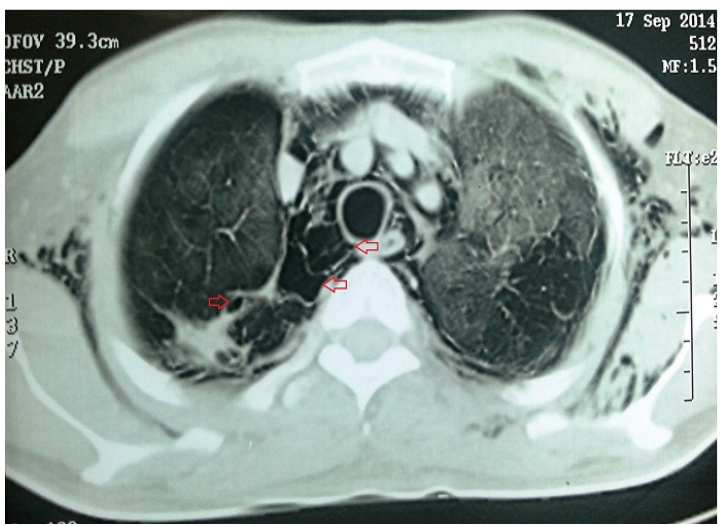

Figure. 4: CECT thorax showing cysts in apices of both lungs (red arrows)

History of Pulmonary Tuberculosis (treated)
$\downarrow$ 7years later
Diagnosed to be seropositive for HIV-1
$\downarrow 3$ weeks later
Presented with shortness of breath, tachypnea.
Diagnosed to be PJP \& treatment started.
$\downarrow 10$ days later
emphysema.
$\downarrow 2$ weeks
Developed pneumomediastinum, pneumothorax \& subcutaneous
Pneumomediastinum, pneumothorax \& subcutaneous emphysema
resolved, anti-retrovirals (ART) initiated \& patient discharged on
co-trimoxazole prophylaxis \& ART.

Figure 5: Timeline of events in evolution of disease.

\section{Discussion:}

Subcutaneous emphysema with pneumomediastinum is a well known complication of blunt or penetrating chest trauma, soft tissue infections or any condition that creates a pressure gradient between the perivascular interstitial and intra-alveolar spaces. Mediastinal emphysema has been described in a variety of clinical situations including parturition ${ }^{6}$, administration of general anaesthesia $^{7}$, acute bronchial asthma $^{8}$ as well as in apparently healthy subjects with no underlying lung disease. 9 Pneumocystis has been associated with pneumatocele and cyst formation in $7-34 \%$ of cases in AIDS patients. ${ }^{10,11,12}$ Pneumomediastinum is rare in AIDS 
patients $^{1}$ infected with Pneumocystis jirovecii and there are no recent reports of this complication.

Several mechanisms have been suggested to explain the pathogenesis of lung cavities in Pneumocystis jirovecii ${ }^{l}$ pneumonia. These include check valve bronchiolar obstruction with distal cyst formation ${ }^{13}$, elastase release from macrophages with subsequent destruction of the alveolar tissue ${ }^{14}$, direct or indirect cytotoxic effects of $\mathrm{HIV}^{15}$ and arterial invasion with thrombosis of the vessels, necrosis and cavitation ${ }^{16}$ among others. Aerosolized Pentamidine used to treat Pneumocystis jirovecii has also been related to pulmonary cysts and pneumothorax ${ }^{17}$ development. Reports also suggest that some of the changes may even be reversible with treatment. ${ }^{18}$ The cysts are typically apical and subpleural, lined by fibrosis and/or alveolar parenchyma with little inflammation in the acute stage. Necrotizing, thin-walled, smaller intraparenchymal cavities lined by organisms, exudate and chronic inflammation can also be seen. ${ }^{19}$ Some propose that the mechanism of spontaneous pneumothorax/pneumomediastinum is spontaneous rupture of necrotic lung tissue occurring in a subgroup of AIDS patients in which the interstitial inflammatory response to Pneumocystis has been accelerated. ${ }^{20}$ Patients at risk of pneumothorax/pneumomediastinum include those with cystic lesions on chest radiograph and those patients with a history of Pneumocystis pneumonia. Patients with AIDS and Pneumocystis pneumonia who develop pneumothorax during mechanical ventilation have a poorer outcome. ${ }^{21}$

In our case, there was no past history of Pneumocystis pneumonia and he is non-smoker. His arm span $(152 \mathrm{~cm})$ is shorter than height $(162 \mathrm{~cm})$ suggesting that he does not have a marfanoid habitus which is a known predisposing factor for developing pneumothorax in young adults. There was no similar history of pneumothorax in his family. Chest radiograph had infiltrative changes in the right lower zone, but no cyst/cavity could be seen and the apices of both lungs appeared normal. However, CECT thorax revealed fibrotic, cystic and bronchiectatic changes. There was also no history of aerosolized Pentamidine therapy. The patient developed pneumomediastinum as well as subcutaneous emphysema while he was recovering. Thus physicians should be alert about this complication in acute as well as in recovery phase.

\section{Conclusion:}

The unique features of our case are as follows: firstly, there was spontaneous pneumomediastinum, pneumothorax and subcutaneous emphysema without any known risk factors as discussed above. Secondly, these complications developed when the patient was in the recovery phase. Treating physicians should therefore have a high index of suspicion while treating patients with Pneumocystis pneumonia and should be vigilant during the whole treatment duration as this potentially fatal but treatable complication can occur anytime during the disease course.

\section{References:}

1. Cheng WL, Ko W-C, Lee N-Y, et-al. Pneumomediastinum in patients with AIDS: a case report and literature review. International Journal of Infectious Diseases 2014; 22:31-34. doi: 10.1016/j.ijid.2013.12.009 
2. Doppman JL., Geelhoed, GW. \& De Vita, VT. Atypical radiographic features in Pneumocystis carinii pneumonia Radiology 1975; 114:39-44.

doi: http://dx.doi.org/10.1148/114.1.39

3. Joe L., Gordin, F. \& Parker, RH. Spontaneous pneumothorax with Pneumocystis carinii infection. Occurrence in patients with acquired immunodeficiency syndrome. Arch Intern Med 1986; 146:1816-1817. doi: 10.1001/archinte.1986.00360210212031

4. Eng RH., Bishburg, E. \& Smith, SM. Evidence for destruction of lung tissues during Pneumocystis carinii infection. Arch Intern Med 1987; 147:746-749. doi: 10.1001/archinte.1987.00370040128022

5. Munsell W.P. Pneumomediastinum: a report of 28 cases and review of the literature. JAMA 1967; 202:129-133. doi: 10.1001/jama.1967.03130210063009

6. Guidelines for the prevention treatment of opportunistic infections in HIV-infected adults and adolescents. 2013. Available from: http://aidsinfo.nih.gov/guidelines

7. Chiles DG., Mannheimer, WN. \& Miller, E. Pneumothorax, pneumomediastinum and massive subcutaneous emphysema after a general anaesthetic. Report of a case J Oral Surg 1970; 28:845-847. No doi

8. Kirsh MM., Orvald TO. Mediastinal and subcutaneous emphysma complicating acute bronchial asthma. Chest 1970; 57:580-581. doi: 10.1378/chest.57.6.580

9. Hamman L. Spontaneous mediastinal emphysema. Bull Johns Hopkins Hosp 1939; 64: 1-21. No doi

10. Chow C, Templeton PA, White CS. Lung cysts associated with Pneumocystis carinii pneumonia: radiographic characteristics, natural history, and complications. American Journal of Roentgenology 1993; 161:527-31. doi: http://dx.doi.org/10.2214/ajr.161.3.8352098

11. Sandhu JS, Goodman PC. Pulmonary cysts associated with Pneumocystis carinii pneumonia in patients with AIDS. Radiology 1989; 173:33-5. doi: 10.1148/radiology.173.1.2789413

12. DeLorenzo LJ, Huang CT, Maguire GP, Stone DJ. Roentgenographic patterns of Pneumocystis carinii pneumonia in 104 patients with AIDS. Chest 1987; 91:323-7. doi: 10.1378/chest.91.3.323

13. Sandhu JS, Goodman PC. Pulmonary cysts associated with Pneumocystis carinii pneumonia in patients with AIDS. Radiology 1989; 173:33-5. doi: 10.1148/radiology.173.1.2789413

14. Eng RH, Bishburg E, Smith SM. Evidence for destruction of lung tissue during Pneumocystis carinii infection. Arch Intern Med 1987; 147:746-9. doi: 10.1001/archinte.147.4.746

15. Kuhlman JE, Knowles MC, Fishman EK, Siegelman SS. Premature bullous pulmonary damage in AIDS: CT diagnosis. Radiology 1989; 173:23-6. doi: 10.1148/radiology.173.1.2781013

16. Liu YC, Thomashefski JF Jr, Tomford JW, Green H. Necrotizing Pneumocystis carinii vasculitis associated with lung necrosis and cavitation in a patient with acquired immunodeficiency syndrome. Arch Pathol Lab Med 1989; 113:494-7. No doi

17. Jules-Elys \&e KM, Stover DE, Zaman MB, et al.. Aerosolized Pentamidine effect on diagnosis and presentation of Pneumocystis carinii pneumonia. Ann Intern Med 1990; 112:750-7. doi: http://dx.doi.org/10.7326/0003-4819-112-10-750

18. Collazos.J, Martinez.E, Mayo.J. Massive reversible pulmonary cysts in a patient with AIDS. Postgrad Med J 1995; 71:753-754, doi:10.1136/pgmj.71.842.753.

19. Feurestein IM, Archer A, Pluda JM, et al. Thin-walled cavities, cysts, and pneumothorax in Pneumocystis carinii pneumonia: further observations with histopathologic correlation. Radiology 1990; 174:697-702. doi: 10.1148/radiology.174.3.2305052 
20. Beers MF, Sohn M, Swartz M. Recurrent pneumothorax in AIDS patients with Pneumocystis pneumonia. A clinicopathologic report of three cases and review of the literature.

Chest. 1990; 98(2):266-70. doi; 10.1378/chest.98.2.266

21. Pastores SM, Garay SM, Naidich DP, Rom WN. Review: pneumothorax in patients with AIDS-related Pneumocystis carinii pneumonia. Am J Med Sci. 1996; 312(5):229-34. doi 10.1097/00000441-199611000-00008 